\title{
GABAergic synaptic transmission regulates calcium influx during spike-timing dependent plasticity
}

\section{Trevor Balena, Brooke A. Acton and Melanie A. Woodin*}

Department of Cell and Systems Biology, University of Toronto, Toronto, ON, Canada

\section{Edited by:}

Per Jesper Sjöström, University

College London, UK

Reviewed by:

David M. Lovinger, National Institutes

of Health, USA

Laura Cancedda, Istituto Italiano di

Tecnologia, Italy

${ }^{*}$ Correspondence:

Melanie A. Woodin, Department of Cell and Systems Biology, University of

Toronto, 25 Harbord Street, Toronto, ON M5S 3G5, Canada.

e-mail:m.woodin@utoronto.ca
Coincident pre- and postsynaptic activity of hippocampal neurons alters the strength of

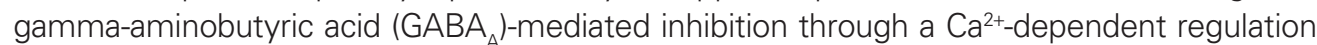
of cation-chloride cotransporters. This long-term synaptic modulation is termed GABAergic spike-timing dependent plasticity (STDP). In the present study, we examined whether the properties of the GABAergic synapses themselves modulate the required postsynaptic $\mathrm{Ca}^{2+}$ influx during GABAergic STDP induction. To do this we first identified GABAergic synapses between cultured hippocampal neurons based on their relatively long decay time constants and their reversal potentials which lay close to the resting membrane potential. GABAergic STDP was then induced by coincidentally $( \pm 1 \mathrm{~ms})$ firing the pre- and postsynaptic neurons at $5 \mathrm{~Hz}$ for $30 \mathrm{~s}$, while postsynaptic $\mathrm{Ca}^{2+}$ was imaged with the $\mathrm{Ca}^{2+}$-sensitive fluorescent dye Fluo4AM. In all cases, the induction of GABAergic STDP increased postsynaptic $\mathrm{Ca}^{2+}$ above resting levels. We further found that the magnitude of this increase correlated with the amplitude and polarity of the GABAergic postsynaptic current (GPSC); hyperpolarizing GPSCs reduced the $\mathrm{Ca}^{2+}$ influx in comparison to both depolarizing GPSCs, and postsynaptic neurons spiked alone. This relationship was influenced by both the driving force for $\mathrm{Cl}^{-}$and $\mathrm{GABA}_{A}$ conductance (which had positive correlations with the $\mathrm{Ca}^{2+}$ influx). The spike-timing order during STDP induction did not influence the correlation between GPSC amplitude and $\mathrm{Ca}^{2+}$ influx, which is likely accounted for by the symmetrical GABAergic STDP window.

Keywords: calcium, chloride, spike-timing dependent plasticity, inhibitory synaptic transmission, hippocampus, KCC2, NKCC1, cation-chloride cotransporter

\section{INTRODUCTION}

GABAergic synaptic transmission can be either excitatory or inhibitory at different stages of nervous system development (Kaila, 1994; Blaesse et al., 2009), or during various pathological states (Kahle et al., 2008). The polarity of GABAergic transmission (hyperpolarizing versus depolarizing) depends on the intracellular concentration of $\mathrm{Cl}^{-}\left(\left[\mathrm{Cl}^{-}\right]_{\mathrm{i}}\right)$; this is because the $\mathrm{GABA}_{\mathrm{A}}$ receptor is a $\mathrm{Cl}^{-}$-permeable ion channel (Kaila, 1994). The $\left[\mathrm{Cl}^{-}\right]_{\mathrm{i}}$ is largely determined by the cation-chloride cotransporters expressed in the neuronal membrane: NKCC1 accumulates $\mathrm{Cl}^{-}$into the neuron (Yamada et al., 2004; Dzhala et al., 2005), while KCC2 transports it out (Rivera et al., 1999). When neuronal $\mathrm{Cl}^{-}$is relatively high due to the dominant expression of NKCC1 during early development, the reversal potential for GABA ( $\left.E_{\mathrm{GABA}} \approx E_{\mathrm{Cl}}\right)$ is depolarized with respect to the resting membrane potential and so GABAergic transmission is depolarizing and sometimes excitatory. In contrast, when neuronal $\mathrm{Cl}^{-}$is low due to the expression of KCC2 in the mature nervous system, $E_{\mathrm{Cl}}$ is hyperpolarized with respect to the resting membrane potential making GABAergic transmission inhibitory.

Recent studies have demonstrated that NKCC1 and KCC2 transporter function is not only regulated by their developmental program, but can also be regulated by neuronal activity (Fiumelli and Woodin, 2007; Blaesse et al., 2009). Coincident pre- and postsynaptic activity at GABAergic synapses results in GABAergic STDP (Woodin et al., 2003; Fiumelli and Woodin, 2007; Balena and Woodin, 2008; Saraga et al., 2008; Xu et al., 2008; Ormond and Woodin, 2009).
Early in development, coincident pre- and postsynaptic activity strengthens inhibition through a $\mathrm{Ca}^{2+}$-dependent regulation of NKCC1, which hyperpolarizes $E_{C 1}$ (Balena and Woodin, 2008). The same pattern of neuronal activity also modifies GABAergic transmission in the mature nervous system through a $\mathrm{Ca}^{2+}$-dependent decrease in KCC2 activity, which depolarizes $E_{\mathrm{CI}}$ (Woodin et al., 2003). The spike-timing window for mature GABAergic inhibition has been characterized as symmetrical. Both positive and negative spike-timing intervals (within $15 \mathrm{~ms} ; \pm 15 \mathrm{~ms}$ ) decrease the strength of inhibition due to $E_{\mathrm{Cl}}$ depolarization (as described above). Noncoincident activity ( $\pm 50 \mathrm{~ms}$ ) also reduces inhibition, but through a decrease in conductance (Woodin et al., 2003).

During GABAergic STDP the $\mathrm{Ca}^{2+}$ influx occurs via voltagegated $\mathrm{Ca}^{2+}$ channels (VGCCs) (Woodin et al., 2003) opened by the back-propagating action potential. When glutamatergic transmission occurs simultaneously with GABAergic STDP induction in hippocampal slices, $\mathrm{Ca}^{2+}$ can also influx via NMDARs (Ormond and Woodin, 2009). Because the opening of VGCCs and NMDARs are voltage-dependent, we hypothesize that GABAergic transmission, which regulates the postsynaptic membrane potential, will regulate the opening of these $\mathrm{Ca}^{2+}$ channel proteins. In turn, this would regulate the $\mathrm{Ca}^{2+}$ influx during GABAergic STDP. It is important to understand how GABAergic synapses regulate $\mathrm{Ca}^{2+}$ influx, because the magnitude of this influx can determine the outcome of plasticity induction (Kano, 1994; Bi and Poo, 1998; Nishiyama et al., 2000; Dan and Poo, 2006). 
We examined $\mathrm{Ca}^{2+}$ dynamics during STDP induction in hippocampal neurons which have formed either depolarizing or hyperpolarizing GABAergic synapses. Using perforated patch-clamp recordings (with gramicidin) and imaging postsynaptic $\mathrm{Ca}^{2+}$ (using Fluo4), we investigated how the polarity and strength of GABAergic transmission regulates $\mathrm{Ca}^{2+}$ influx. We further analyzed this relationship by examining several aspects of GABAergic transmission, including $\mathrm{GABA}_{\mathrm{A}}$ conductance, $E_{\mathrm{Cl}}$, and $\mathrm{Cl}^{-}$driving force. We found that $\mathrm{GABA}_{\mathrm{A}}$-mediated transmission regulates $\mathrm{Ca}^{2+}$ influx during the induction of STDP, with the strength of the synapse significantly altering the magnitude of the postsynaptic $\mathrm{Ca}^{2+}$ influx in a linear fashion.

\section{MATERIALS AND METHODS HIPPOCAMPAL CULTURES}

Low-density cultures of dissociated embryonic rat hippocampal neurons were prepared as previously described (Balena and Woodin, 2008). In brief, embryonic day 18 (E18) pregnant Sprague-Dawley rats were briefly exposed to carbon dioxide and cervically dislocated in accordance with guidelines from the University of Toronto Animal Care Committee and the Canadian Council on Animal Care. Hippocampi were then removed and treated with trypsin for $15 \mathrm{~min}$ at $37^{\circ} \mathrm{C}$, followed by gentle trituration. The dissociated cells were plated at a density of 50,000 cells/mL on poly-L-lysine coated $25 \mathrm{~mm}$ glass coverslips (in $35 \mathrm{~mm}$ Petri dishes). Cells were plated in Neurobasal medium (Invitrogen, Carlsbad, California, USA), supplemented with 2\% B-27 (Invitrogen, Carlsbad, California, USA). Twenty-four hours after plating, half of the medium was replaced with the original plating medium containing $20 \mathrm{mM} \mathrm{KCl}$. Forty-eight hours after plating, and every 3 days following, one third of the medium was replaced with DMEM (Invitrogen, Carlsbad, California, USA) supplemented with 10\% heat-inactivated fetal bovine serum (Invitrogen, Carlsbad, California, USA), 10\% Ham's F12 with L-glutamine (Invitrogen, Carlsbad, California, USA), 1\% penicillin-streptomycin (SigmaAdrich, Oakville, Ontario, Canada), $10 \mathrm{mM} \mathrm{KCl}$ and $15 \mathrm{mM}$ HEPES. Both glia and neurons were present under these culture conditions. Cells were recorded from after 8-13 days in culture.

\section{ELECTROPHYSIOLOGY}

Whole-cell perforated patch recordings using gramicidin $(50 \mu \mathrm{g} /$ $\mathrm{mL}$; Sigma-Adrich, Oakville, Ontario, Canada) were performed on pairs of synaptically connected cultured hippocampal neurons. The recording pipettes were made from glass capillaries (World Precision Instruments Inc., Sarasota, Florida, USA), with a resistance of 4-10 M $\Omega$. The pipettes were filled with an internal solution containing $150 \mathrm{mM} \mathrm{KCl}, 10 \mathrm{mM}$ HEPES, and gramicidin, $\mathrm{pH}$ 7.4, osmolarity $=300 \mathrm{mOsmol}$. The cultures were continuously perfused (approximately $1 \mathrm{~mL} / \mathrm{min}$ ) with extracellular recording solution containing (in mM): $150 \mathrm{NaCl}, 3 \mathrm{KCl}, 3 \mathrm{CaCl}_{2} \cdot 2 \mathrm{H}_{2} \mathrm{O}, 2 \mathrm{MgCl}_{2} \cdot 6 \mathrm{H}_{2} \mathrm{O}$, 10 HEPES, 5 Glucose, pH 7.4, osmolarity = 307-315 mOsmol. Recordings were performed with a MultiClamp 700B (Molecular Devices Inc., Sunnyvale, California, USA) patch-clamp amplifier. Signals were filtered at $5 \mathrm{kHz}$ using amplifier circuitry. Data was acquired and analyzed using Clampfit 9 (Molecular Devices Inc., Sunnyvale, California, USA). Recordings started after the series resistance had dropped below $30 \mathrm{M} \Omega$. For assaying synaptic connectivity, each neuron was stimulated at a low frequency $(0.05 \mathrm{~Hz})$ by a $1 \mathrm{~ms}$ step depolarization from -70 to $+20 \mathrm{mV}$ in voltage-clamp mode. GPSCs were distinguishable from excitatory postsynaptic currents (EPSCs) by longer decay times. Upon occasion we did detect autaptic GABAergic synapses in our cultures, however we did not examine these synapses in the present study. During the STDP induction protocol both neurons were switched to current-clamp mode and injected with current (minimal stimulation, $2 \mathrm{~ms}$ ) both pre- and postsynaptically to generate an action potential in each cell at a frequency of $5 \mathrm{~Hz}$ for $30 \mathrm{~s}$. The interval between spike induction was $\pm 5 \mathrm{~ms}$, which resulted in a spike-timing interval of $\pm 1 \mathrm{~ms}$ between onset of the GABAergic postsynaptic potential (GPSP) and the postsynaptic action potential. This protocol resulted in 150 pairs of pre- and postsynaptic action potentials. All recordings were performed at room temperature $\left(25^{\circ} \mathrm{C}\right)$.

The resting membrane potential was determined in currentclamp mode in the absence of current injection or synaptic activity. $E_{\mathrm{Cl}}$ was determined by varying the holding potential of the postsynaptic cell in $10 \mathrm{mV}$ increments and measuring the resulting GPSC amplitude; each set of current-voltage (I-V) measurements was repeated after a 5-min interval. A linear regression of both sets of GPSC amplitude measurements was then used to calculate the voltage dependence of GPSCs. The intercept of this line with the abscissa was taken as $E_{\mathrm{Cl}}$. The slope of the same line was taken as GPSC conductance. The difference between the resting membrane potential and $E_{\mathrm{Cl}}$ was taken as the driving force.

$\mathrm{GABA}_{\mathrm{A}}$ receptors are permeable to both $\mathrm{HCO}_{3}{ }^{-}$and $\mathrm{Cl}^{-}(\sim 0.2-0.4$ ratio; Kaila, 1994). Due to the relatively positive $\mathrm{HCO}_{3}^{-}$equilibrium potential $(\sim-10 \mathrm{mV})$, which is set by mechanisms that control intracellular $\mathrm{pH}$ regulation (Kaila and Voipio, 1987), $\mathrm{HCO}_{3}^{-}$mediates an inward, depolarizing current (Kaila and Voipio, 1987; Kaila et al., 1993; Gulledge and Stuart, 2003). However, our experiments were performed in bicarbonate-free solution buffered with HEPES, and thus $\mathrm{GABA}_{\mathrm{A}}$ receptor activation was solely mediating a $\mathrm{Cl}^{-}$current. For this reason we report $E_{\mathrm{Cl}}$ and not $E_{\mathrm{GABA}}$.

\section{FLUORESCENCE IMAGING}

To assess the effect of STDP induction on postsynaptic $\mathrm{Ca}^{2+}$ influx the hippocampal neurons were loaded with the membrane-permeable fluorescent $\mathrm{Ca}^{2+}$ indicator Fluo4-AM (Invitrogen; Carlsbad, CA, USA) for $30 \mathrm{~min}$ at $37^{\circ} \mathrm{C}, 5 \% \mathrm{CO}_{2}$. The Fluo4 was dissolved in dimethyl sulfoxide (DMSO) and 20\% pluronic acid to a stock concentration of $1 \mathrm{mM}$ and then diluted to $1 \mu \mathrm{M}$ in our extracellular recording solution. Following dye-loading the cells were thoroughly washed with extracellular recording solution. Cells were then transferred to the recording chamber of an inverted microscope (Olympus IX71) equipped with an Olympus $0.6 \mathrm{NA} \times 40$ objective. Fluo4 was excited at $488 \mathrm{~nm}$ through a monochromator (Photon Technology International (Canada) Inc., London, ON), controlled by the ImageMaster software (Photon Technology International (Canada) Inc., London, ON). Fluorescence emission of labeled cells at $510 \mathrm{~nm}$ was detected with a 16-bit CCD camera (Cascade 650, Photometrics, Roper Scientific, Tuscon, AZ, USA). Images of $653 \times 492$ pixels were accumulated at 500-1000 ms intervals.

\section{FLUORESCENCE ANALYSIS}

Analysis of the fluorescence signals was performed off-line on the image sequences as they were originally acquired. Analysis was performed on regions of interest (ROIs) that encompassed 
approximately $80 \%$ of the soma (which ranged from $10-50 \mu \mathrm{M}$ in diameter). Fluorescence was plotted against time to yield a graph of the fluorescence changes over the STDP induction period. $F_{0}$ was taken to be the fluorescence from the last image before induction began. $F_{\text {peak }}$ was taken to be the maximum fluorescence level reached over the course of the induction. $F_{30}$ was taken to be the fluorescence from the final image during the induction period. $\Delta F_{30}$ was calculated as the percentage difference between $F_{0}$ and $F_{30} ; \Delta F_{\text {peak }}$ was calculated as the percentage difference between $F_{0}$ and $F_{\text {peak }}$. The area under the graph was normalized to $F_{0}$ to provide $F_{\text {area }}$. We determined that photobleaching did not impact our $\mathrm{Ca}^{2+}$ analysis. This determination was made by comparing the fluorescence of quiescent cells during the first and last $5 \mathrm{~s}$ of a 30-s image acquisition and finding no significant difference in fluorescence $(p=0.06)$. Thus, we did not alter the image analysis further to account for photobleaching.

\section{STATISTICAL ANALYSIS}

All data are presented as mean \pm SEM. Linear regression analysis was used to obtain correlation coefficients and corresponding $p$-values. One-way ANOVA was used to compare the $F_{\text {area }}$ values of depolarizing and hyperpolarizing synapses to those of neurons fired alone (post only). Paired $t$-tests were used to compare $E_{\mathrm{Cl}}$ and resting membrane potential values when those values were obtained from the same neurons. All other statistical analysis used unpaired $t$-tests. All statistical analysis was performed using SigmaStat 2.03.

\section{RESULTS}

\section{CHARACTERIZATION AND LOCALIZATION OF GABAergic SYNAPSES}

In order to examine $\mathrm{Ca}^{2+}$ influx during STDP induction, we first had to locate synaptically connected neurons and characterize the synapse between them. We did this using dual perforated patchclamp recordings from pairs of hippocampal neurons cultured at a low density. Hippocampal cultures were prepared from E18 rats and recorded from after 8-13 days in culture; they contained glia, pyramidal neurons and GABAergic interneurons. Synaptic connections were identified by stimulating one neuron and monitoring for the presence of postsynaptic currents in the other. After we located a synapse, our first indication that it was GABAergic came from the relatively long time course of the GPSC in voltage-clamp mode $(35.50 \pm 3.37 \mathrm{~ms}$ for GPSCs, as opposed to $5.76 \pm 0.64 \mathrm{~ms}$ for glutamatergic currents; Balena and Woodin, 2008). If the synapse had a time course consistent with GABAergic currents we then determined the $E_{\mathrm{Cl}}$ by constructing an $\mathrm{I}-\mathrm{V}$ curve; the intersection of the curve with the $x$-axis was taken to be $E_{\mathrm{Cl}}$ (Figures 1A,B). Because the extracellular recording solution was free of $\mathrm{HCO}_{3}^{-}, E_{\mathrm{Cl}} \approx E_{\mathrm{GABA}}$. Based on the relation of $E_{\mathrm{Cl}}$ to resting membrane potential, we characterized GABAergic synapses as either: (1) depolarizing, when $E_{\mathrm{Cl}}$ was more positive than the resting membrane potential (Figure 1A); or (2) hyperpolarizing, when $E_{\mathrm{Cl}}$ was more negative than the resting membrane potential (Figure 1B). Depolarizing GABAergic synapses had an average $E_{\mathrm{Cl}}$ of $-58.33 \pm 2.63 \mathrm{mV}$, which was significantly different from the resting membrane potential of those neurons $(-70.33 \pm 1.17 \mathrm{mV} ; n=12$; paired $t$-test $p<0.001$; Figure 1C). In all cases $E_{\mathrm{Cl}}$ was hyperpolarizing with respect to action potential threshold, and thus depolarizing synapses were not excitatory. Hyperpolarizing synapses had an average $E_{\mathrm{Cl}}$ of $-75.87 \pm 1.74 \mathrm{mV}$, which was significantly different from
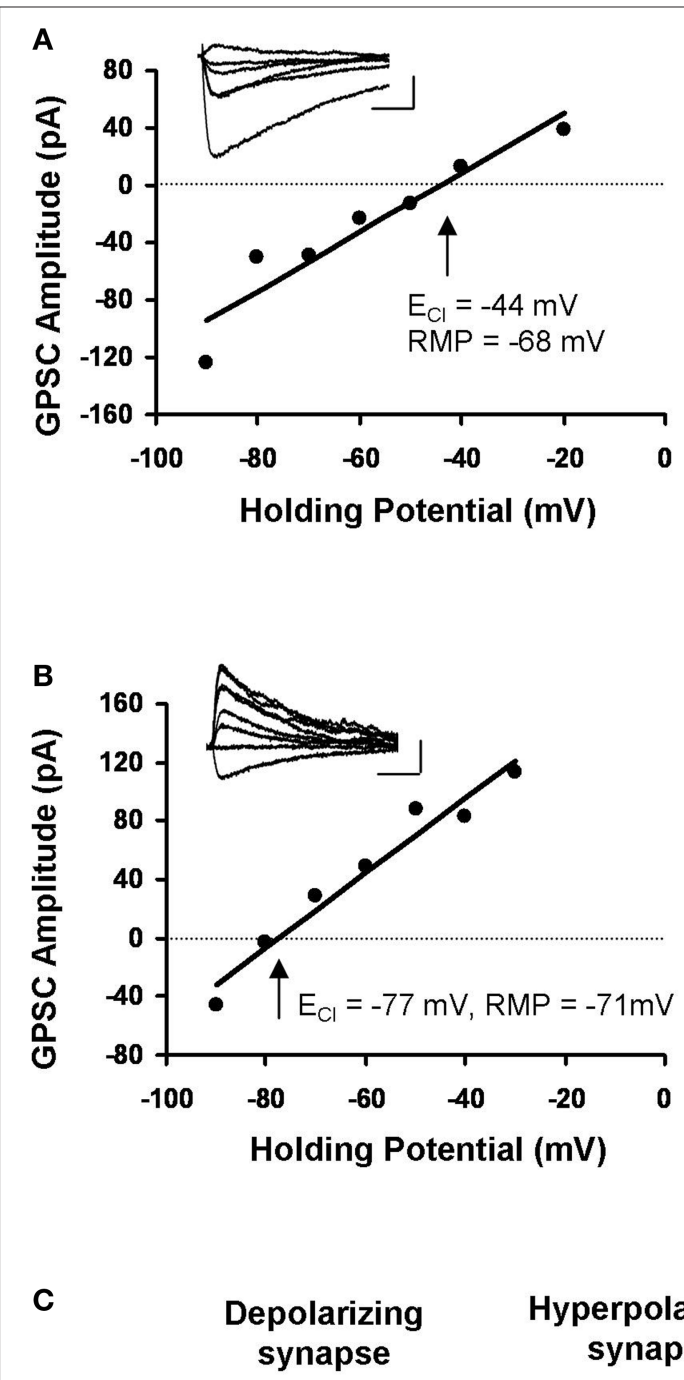

\section{Hyperpolarizing synapse}

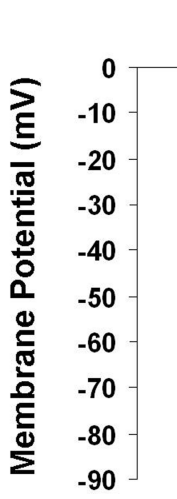

$\mathrm{E}_{\mathrm{Cl}} \quad \mathrm{RMP}$

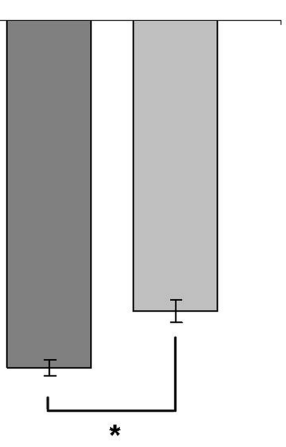

FIGURE 1 | Characterization of GABAergic synapses. (A) Example of an I-V curve from a depolarizing GABAergic synapse. The postsynaptic membrane potential was stepped in $10 \mathrm{mV}$ increments while stimulating GABAergic synapses to generate the I-V curve. The holding potential at which the GPSC amplitude was $0 \mathrm{mV}$ (dashed line) was taken as $E_{\mathrm{Cl}}$. Inset: sample traces of GPSCs recorded during the construction of the I-V curve. Legend: $20 \mathrm{~ms}$, $45 \mathrm{mV}$. (B) Example of an I-V curve from a hyperpolarizing GABAergic synapse. Characterized as in (A). Legend: $20 \mathrm{~ms}, 45 \mathrm{mV}$. (C) The average $\pm S E M$ for $E_{C l}$ and resting membrane potential (RMP) at depolarizing and hyperpolarizing synapses. ${ }^{*}$ indicates statistical significance. 
their resting membrane potential values $(-63.40 \pm 2.44 \mathrm{mV} ; n=10$; paired $t$-test $p<0.001$; Figure 1C). There was a significant difference between the $E_{\mathrm{Cl}}$ values $(p<0.001)$ and resting membrane potential values ( $p=0.014$ ) of depolarizing and hyperpolarizing synapses. The slope of each $\mathrm{I}-\mathrm{V}$ curve provided the $\mathrm{GABA}_{\mathrm{A}}$ conductance of that synapse. Depolarizing GABAergic synapses had a conductance of $1.85 \pm 0.34 \mathrm{pS}(n=12)$, and hyperpolarizing synapses had a conductance of $1.74 \pm 0.35 \mathrm{pS}(n=10)$; there was no significant difference between the conductances of the two populations $(p=0.824)$.

\section{SIMULTANEOUS STDP INDUCTION AND IMAGING OF CA ${ }^{2+}$ DYNAMICS}

Following characterization of the GABAergic synapse we examined the $\mathrm{Ca}^{2+}$ dynamics during the induction of STDP. To do this, we loaded neurons with the $\mathrm{Ca}^{2+}$-sensitive cell permeant fluorescent dye Fluo4-AM (Figures 2A,B). A sequence of fluorescence images was then acquired at $1-2 \mathrm{~Hz}$ during STDP induction. GABAergic STDP was induced in current-clamp mode; both the pre- and postsynaptic neurons were induced to fire action potentials (using minimal stimulation) at a frequency of $5 \mathrm{~Hz}$ for $30 \mathrm{~s}$. The interval between spike induction was $\pm 5 \mathrm{~ms}$, which resulted in a spiketiming interval of $\pm 1 \mathrm{~ms}$ between onset of the GPSP and the postsynaptic action potential. This yielded a graph representing the changes in Fluo4 fluorescence (as a measure of $\mathrm{Ca}^{2+}$ ) over time (Figure 2C). This data was normalized to the baseline fluorescence level and expressed as a percentage increase. We analyzed three measures of the change in fluorescence during STDP induction: (1) $\Delta F_{30}(\%)$, the change in fluorescence from $F_{0}$ to $F_{30} ;(2) \Delta F_{\text {peak }}(\%)$, the change in fluorescence from $F_{0}$ to $F_{\text {peak }}$; and (3) $F_{\text {area }}$, the area
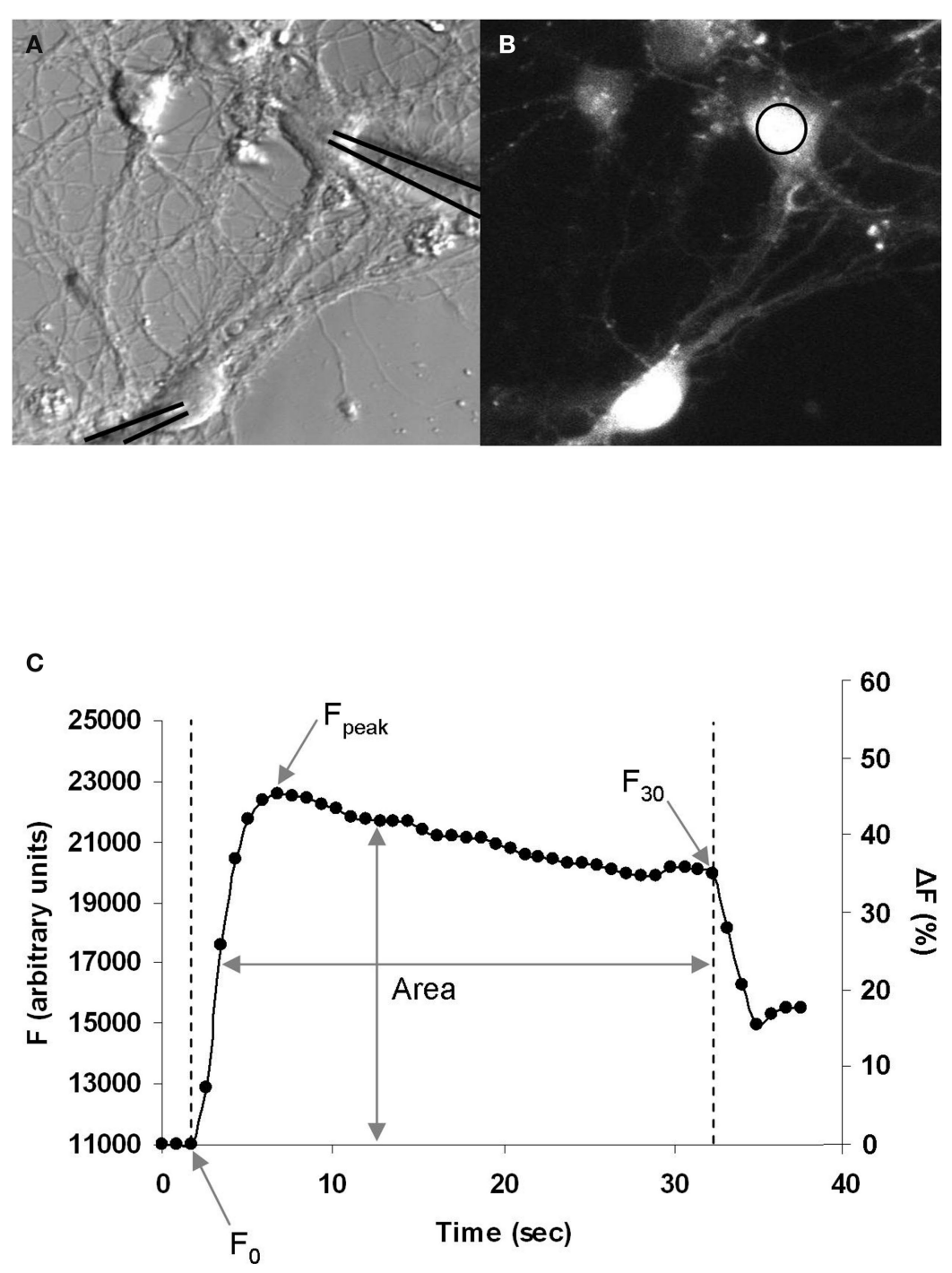

FIGURE 2 | Postsynaptic $\mathrm{Ca}^{2+}$ imaging during GABAergic STDP induction. (A) Bright field image of neurons during recording. The recording pipettes have been overlaid with black lines to clearly indicate the neurons being recorded. (B) Fluo4AM fluorescence image of the same neurons as in (A), measuring changes in $\mathrm{Ca}^{2+}$ during STDP induction. $\mathrm{Ca}^{2+}$ was measured in the area of interest on the soma (circle). (C) Example graph of the increase in Fluo4 fluorescence during induction, which begins at $F_{0}$ with the onset of stimulation, peaks at $F_{\text {peak }}$ and ends at $F_{30}$ after $30 \mathrm{~s}$ of stimulation. The area of under the curve over this time period was used to calculate $F_{\text {area }}$ which normalizes the area to $F_{0}$. Left axis: arbitrary fluorescence units. Right axis: the same graph expressed as a percentage increase from baseline. 
under the curve normalized to $F_{0}$. Increasing the image acquisition frequency to 100-200 ms intervals did not significantly change the $\Delta F_{\text {peak }}$ for depolarizing GABAergic synapses $(p=0.898)$, indicating that the standard acquisition rate was sufficient to resolve the peak of the $\mathrm{Ca}^{2+}$ fluorescence. We chose to exclusively examine the $\mathrm{Ca}^{2+}$ influx at the soma of the postsynaptic neurons for two reasons. First, the majority of GABAergic neurons innervate the proximal dendrites and soma of postsynaptic neurons (Cobb et al., 1995; Freund and Buzsaki, 1996; Di Cristo et al., 2004; Huang, 2006). Second, we recently demonstrated that GABAergic STDP is induced by feed-forward interneurons which target the soma of pyramidal neurons in the CA1 region of the hippocampus (Ormond and Woodin, 2009).

\section{GPSC POLARITY DETERMINES THE MAGNITUDE OF CA ${ }^{2+}$ INFLUX DURING STDP INDUCTION}

$\mathrm{Ca}^{2+}$ influx is required for GABAergic STDP (Woodin et al., 2003), but whether or not the dynamics of the influx depend on the properties of the synapse had not yet been determined. After recording from a population of neurons that included both depolarizing and hyperpolarizing GPSCs and imaging their $\mathrm{Ca}^{2+}$ dynamics, we examined the relationship between GPSC amplitude and $\mathrm{Ca}^{2+}$ influx during STDP induction. GPSC amplitude correlated strongly with $\mathrm{Ca}^{2+}$ influx $\left(F_{\text {area }}\right)$; the linear regression analysis yielded similarly high $r$-squared value regardless of the spike-timing order [pre/post $r$-squared $=0.66(p=0.002)$, post/ pre r-squared $=0.65(p=0.003)$, all synapses $r$-squared $=0.65$ $(p<0.001)$; Figure 3A]. GPSC amplitude also correlated strongly with other measure of $\mathrm{Ca}^{2+}$ influx, $\Delta F_{30}$ [pre/post r-squared $=0.65$ $(p=0.002)$, post/pre $r$-squared $=0.60(p=0.005)$, all synapses r-squared $=0.62(p<0.001)]$ and $\Delta F_{\text {peak }}[$ pre/post r-squared $=0.62$ $(p=0.004)$, post/pre $r$-squared $=0.63(p=0.004)$, all synapses r-squared $=0.62(p<0.001)]$. Thus, regardless of the fluorescence measure examined, there was a strong relationship between $\mathrm{Ca}^{2+}$ influx and the nature of the GABAergic synapse; depolarizing synapses correlated with large increases in $\mathrm{Ca}^{2+}$, while hyperpolarizing synapses correlated with smaller increases.

The correlation between $\mathrm{Ca}^{2+}$ influx and GPSC amplitude was further quantified by comparing the fluorescence increase during STDP induction between all depolarizing synapses $\left(E_{\mathrm{Cl}}=-58.33 \pm 2.63 \mathrm{mV} ; n=12\right)$, all hyperpolarizing synapses $\left(E_{\mathrm{Cl}}=-75.87 \pm 1.74 \mathrm{mV} ; n=10\right)$, and neurons with no synapses (which we call "post only"; $n=11$ ). The post only neurons were also stimulated at $5 \mathrm{~Hz}$ for $30 \mathrm{~s}$. There were significant differences between the $\mathrm{Ca}^{2+}$ influx at depolarizing and hyperpolarizing synapses regardless of the measure analyzed $\left(F_{\text {area }} p=0.004 ; F_{\text {peak }}\right.$ $p=0.005 ; F_{30} p=0.004$; Figure $\left.3 \mathrm{~B}\right)$. $\mathrm{Ca}^{2+}$ influx at hyperpolarizing synapses were also significantly different from the influx at post only neurons that fired in the absence of a synapse when $F_{\text {area }}$ and $F_{30}$ were analyzed ( $p=0.04$ and $p=0.048$, respectively); however when $F_{\text {peak }}$ was analyzed there was not a significant difference between hyperpolarizing synapses and post only neurons $(p=0.06)$. Thus, we can conclude that depolarizing neurons let in the same amount of $\mathrm{Ca}^{2+}$ during STDP induction as the postsynaptic neurons spiking alone (independent of a synapse). However, when the GPSC becomes hyperpolarizing it has a strong ability to decrease the $\mathrm{Ca}^{2+}$ influx.
At glutamatergic synapses the order of spiking (pre/post versus post/pre) during STDP induction determines the polarity of the plasticity (LTP versus LTD, respectively; Bi and Poo, 1998). However, at GABAergic synapses the order of spiking during induction does not determine the nature of plasticity (as evidenced by the symmetrical spike-timing window Woodin et al., 2003). We thus hypothesized that $\mathrm{Ca}^{2+}$ influx during STDP induction should be independent of the spike-timing order. We found no significant difference in $\mathrm{Ca}^{2+}$ influx $\left(F_{\text {area }}\right)$ between synapses induced with positive or negative spiketiming intervals; this was true for both depolarizing $(p=0.96)$ and hyperpolarizing synapses ( $p=0.77$; Figure $3 \mathrm{C}$ ). We also found no significant difference between the time taken for the $\mathrm{Ca}^{2+}$ influx to reach its maximum between pre/post and post/pre synapses $(p=0.968)$.

\section{GABAergic SYNAPSE PROPERTIES DETERMINE CA ${ }^{2+}$ DYNAMICS DURING STDP INDUCTION}

The $\mathrm{Ca}^{2+}$ influx during STDP induction was affected by GPSC amplitude. GPSC amplitude is determined by both $E_{\mathrm{Cl}}$ and $\mathrm{GABA}_{\mathrm{A}}$ conductance (Kaila, 1994); thus we asked which of these properties was the most influential in regulating postsynaptic $\mathrm{Ca}^{2+}$. We found that the linear regression of $E_{\mathrm{Cl}}$ versus $\mathrm{Ca}^{2+}$ influx $\left(F_{\text {area }}\right)$ yielded low r-squared values $[0.31(p=0.078)$ for pre/post synapses, $0.25(p=0.114)$ for post/pre synapses, and 0.26 ( $p=0.015$ ) for all synapses] (Figure $4 \mathrm{~A}$ ). Low $\mathrm{r}$-squared values indicate that the trend line does not accurately predict the relationship between $E_{\mathrm{Cl}}$ and $\mathrm{Ca}^{2+}$ influx. Thus, the difference between the slopes of the trend lines for pre/post and post/pre synapses does not necessarily indicate that the spike-timing order influences the $\mathrm{Ca}^{2+}$ influx. $E_{\mathrm{Cl}}$ also did not correlate strongly with $\Delta F_{30}$ [pre/post r-squared $=0.26(p=0.114)$, post/pre r-squared $=0.27$ $(p=0.104)$, all synapses $\mathrm{r}$-squared $=0.26(p=0.017)]$ or $\Delta F_{\text {peak }}$ [pre/post r-squared $=0.29(p=0.086)$, post/pre $\mathrm{r}$-squared $=0.25$ $(p=0.119)$, all synapses $r$-squared $=0.26(p=0.016)]$.

Perhaps a more useful measure when considering the $\mathrm{Cl}^{-}$gradient is the driving force for $\mathrm{Cl}^{-}$, which is the difference between the membrane potential and $E_{\mathrm{Cl}}$. We found that the driving force correlated more strongly with $\mathrm{Ca}^{2+}$ influx $\left(F_{\text {area }}\right)$ than $\operatorname{did} E_{\mathrm{Cl}}$ alone, with r-squared values of 0.39 ( $p=0.041)$ for pre/post synapses, 0.31 ( $p=0.076)$ for post/ pre synapses, and $0.34(p=0.004)$ for all synapses (Figure 4B). Other measures of $\mathrm{Ca}^{2+}$ influx, $\Delta F_{30}$ [pre/post r-squared $=0.42(p=0.032)$, post/pre r-squared $=0.33(p=0.063)$, all synapses $r$-squared $=0.37$ $(p=0.003)]$ and $\Delta F_{\text {peak }}[$ pre/post r-squared $=0.35(p=0.055)$, post $/$ pre r-squared $=0.33(p=0.066)$, all synapses $r$-squared $=0.33(p=0.005)]$, provided similar results as $F_{\text {area }}$.

$\mathrm{GABA}_{\mathrm{A}}$ conductance was found to correlate with $F_{\text {area }}$ to a higher degree than did $E_{\mathrm{Cl}}$ or driving force, with r-squared values of $0.57(p=0.007)$ for pre/post synapses, $0.68(p=0.002)$ for post/pre synapses, and $0.62(p<0.001)$ for all synapses (Figure 4C). Conductance also correlated well with $\Delta F_{30}$ [pre/ post $\mathrm{r}$-squared $=0.52(p=0.013)$, post $/$ pre $\mathrm{r}$-squared $=0.62$ $(p=0.004)$, all synapses r-squared $=0.56(p<0.001)]$ and $\Delta F_{\text {peak }}$ [pre/post r-squared $=0.52(p=0.012)$, post/pre $r$-squared $=0.66$ $(p=0.003$, all synapses $r$-squared $=0.58(p<0.001)]$.

\section{DISCUSSION}

At GABAergic synapses, the induction of STDP requires an increase in postsynaptic $\mathrm{Ca}^{2+}$ (Woodin et al., 2003; Xu et al., 2008; Ormond and Woodin, 2009). Depending on the stage of nervous system 


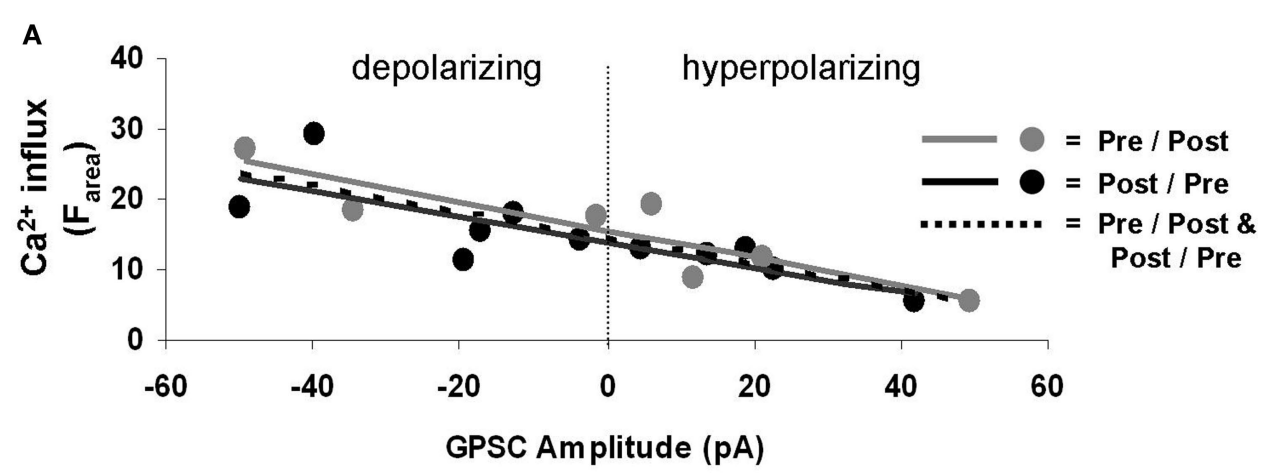

B

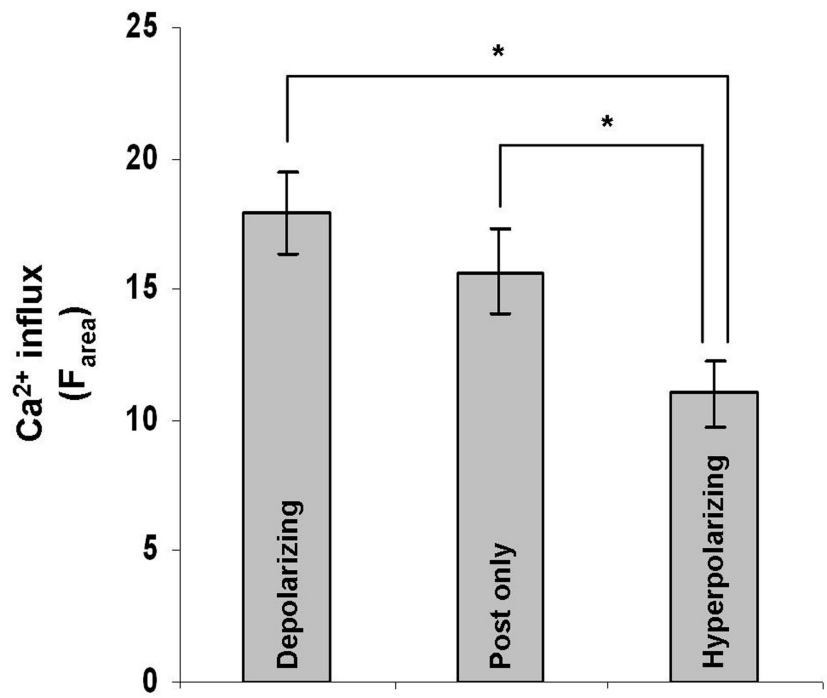

C

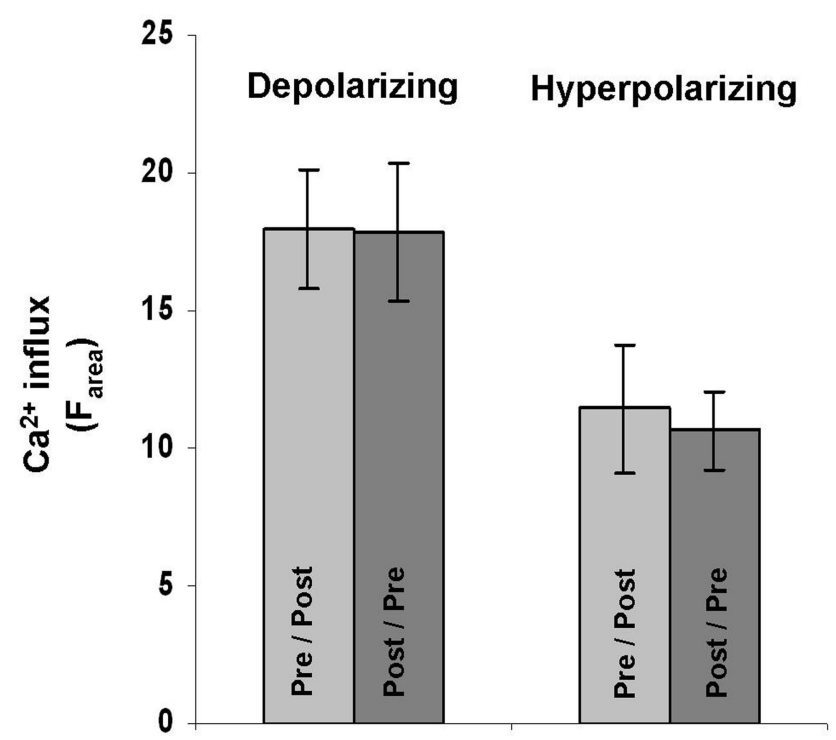

FIGURE 3 | GPSC amplitude affects postsynaptic $\mathrm{Ca}^{2+}$ dynamics during STDP induction. (A) GPSC amplitude strongly correlates with $\mathrm{Ca}^{2+}$ influx regardless of the order of spike-timing. (B) Depolarizing synapses result in a significantly larger $\mathrm{Ca}^{2+}$ influx during STDP induction than do hyperpolarizing synapses $(p=0.004)$. Hyperpolarizing synapses also result in a significantly lower $\mathrm{Ca}^{2+}$ influx than the firing of a neuron alone (post only; $p=0.045$ ). (C) There was no significant difference in $\mathrm{Ca}^{2+}$ influx when STDP was induced in post/pre or pre/ post orders at depolarizing $(p=0.96)$ and hyperpolarizing synapses $(p=0.77)$. 
A

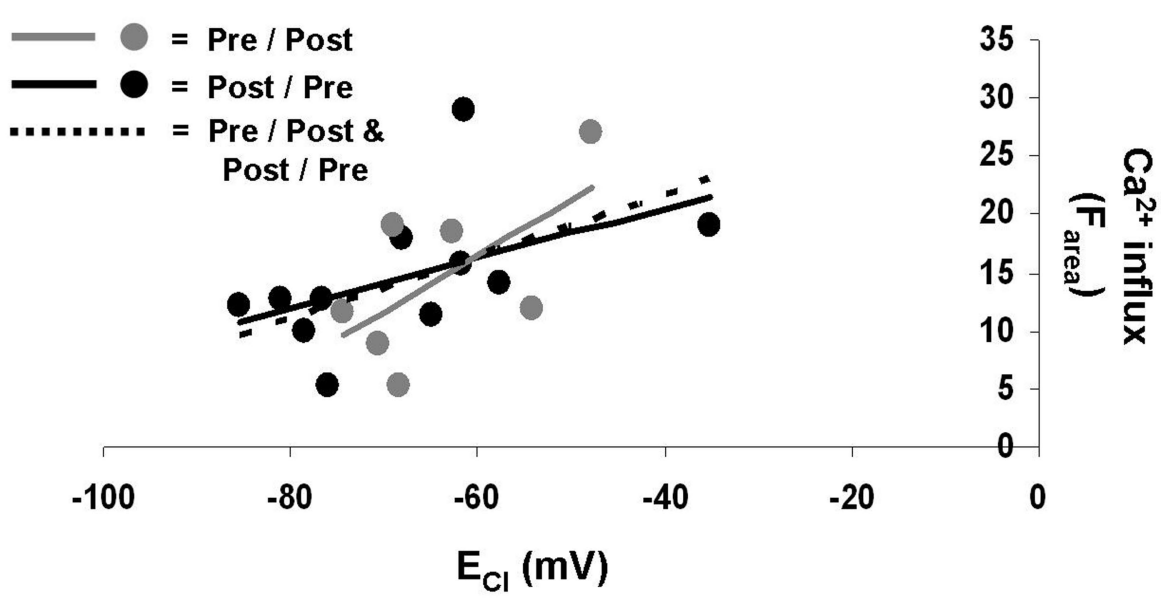

B

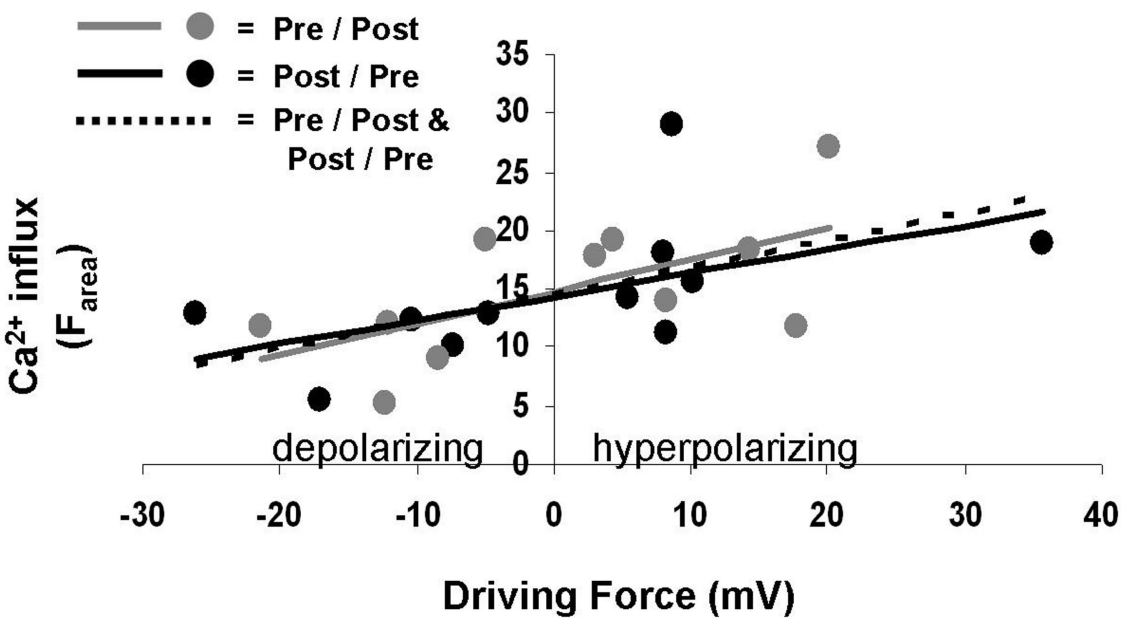

C

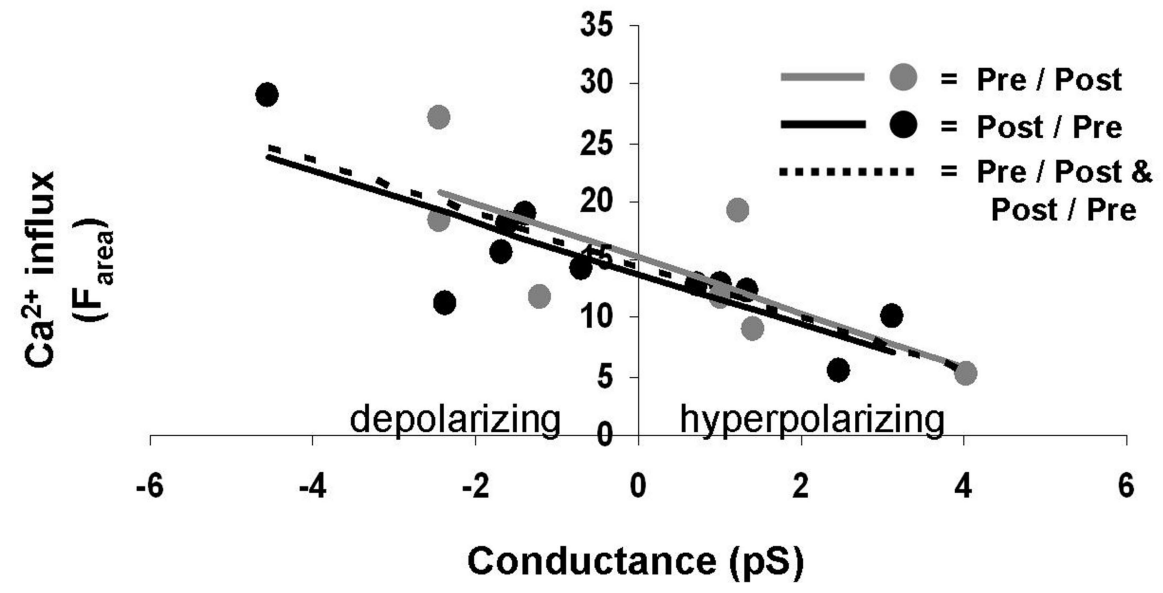

FIGURE $4 \mid E_{\mathrm{Cl}}$, driving force, and $\mathrm{GABA} \mathrm{A}_{\mathrm{A}}$ conductance influence $\mathrm{Ca}^{2+}$ influx during STDP induction. (A) $E_{\mathrm{Cl}}$ does not correlate well with $\mathrm{Ca}^{2+}$ influx. Gray points and solid gray line represent pre/post synapses. Black points and solid black line indicate post/pre synapses. Dotted line indicates all synapses combined. (B) Driving force correlates better with $\mathrm{Ca}^{2+}$ influx. Gray points and solid gray line represent pre/post synapses. Black points and solid black line indicate post/pre synapses. Dotted line indicates all synapses combined. (C) $\mathrm{GABA}_{\mathrm{A}}$ conductance also correlates well with $\mathrm{Ca}^{2+}$ influx. Gray points and solid gray line represent pre/post synapses. Black points and solid black line indicate post/pre synapses. Dotted line indicates all synapses combined. 
development GABAergic synapses can be either depolarizing or hyperpolarizing, which led us to hypothesize that the nature of GABAergic transmission regulates the magnitude of the $\mathrm{Ca}^{2+}$ influx. Our present results found this hypothesis to be true; depolarizing GABAergic synapses characteristic of immature neuronal circuits produced larger $\mathrm{Ca}^{2+}$ influxes during STDP induction than hyperpolarizing GABAergic synapses, which are more commonly found in the mature central nervous system. Our analysis further revealed that this relationship between GABAergic synapses and $\mathrm{Ca}^{2+}$ influx can be accounted for by the two main properties of GABAergic synapses (the driving force for $\mathrm{Cl}^{-}$and the $\mathrm{GABA}_{\mathrm{A}}$ receptor conductance) but did not depend on the order of spike timing.

It is already well known that synaptic transmission contributes to the postsynaptic $\mathrm{Ca}^{2+}$ influx during glutamatergic STDP induction (Markram et al., 1997; Bi and Poo, 1998, 2001; Debanne et al., 1998; Zhang et al., 1998). At these excitatory synapses, $\mathrm{Ca}^{2+}$ influx occurs primarily via VGCCs opened by the back-propagating action potential, and via NMDARs opened by the coincident occurrence of the back-propagating action potential and postsynaptic glutamate binding. How can our understanding of the $\mathrm{Ca}^{2+}$ influx during glutamatergic STDP induction be related to GABAergic STDP? It depends on the polarity of the GABAergic synapse. When $E_{\mathrm{Cl}}$ sits below the resting membrane potential the GPSC will hyperpolarize the postsynaptic membrane during STDP induction, resulting in a smaller $\mathrm{Ca}^{2+}$ influx than if that same postsynaptic neuron was spiking alone (at $5 \mathrm{~Hz}$ for $30 \mathrm{~s}$, in the absence of synaptic transmission). This smaller $\mathrm{Ca}^{2+}$ influx presumably results from hyperpolarizing GPSCs decreases the opening of VGCCs (which are activated by action potential firing during STDP induction). However, at depolarizing GABAergic synapses, the $\mathrm{Ca}^{2+}$ influx during STDP induction for both positive and negative spike-timing intervals does not differ significantly from when the postsynaptic neuron spikes alone. This indicates that the additional depolarization is insufficient to open more VGCCs, either because the majority of available VGCCs have already been opened by the action potential, or because the magnitude of depolarization is not sufficient to open VGCCs.

We already know that the required $\mathrm{Ca}^{2+}$ influx during GABAergic STDP occurs partly through L-type VGCCs (Woodin et al., 2003; Ormond and Woodin, 2009). However, this cannot be the only source of $\mathrm{Ca}^{2+}$ influx because when GABAergic synapses are blocked or absent (post only) the same spiking pattern which also opens VGCCs fails to induce plasticity. This indicates that there are either additional sources of $\mathrm{Ca}^{2+}$ influx required for STDP, or that a component of the GABAergic signaling combines with the L-type $\mathrm{Ca}^{2+}$ influx to induce plasticity. We have preliminary evidence for the involvement of T-type VGCCs during hyperpolarizing GABAergic STDP (Balena and Woodin, 2009); these channels require membrane hyperpolarization to be removed from their inactive state but also require subsequent membrane depolarization to become activated (Magee et al., 1995; Perez-Reyes and Lory, 2006). Thus during hyperpolarizing GABAergic transmission we believe that the $\mathrm{Ca}^{2+}$ influx occurs both through L-type VGCCs (which have a reduced opening compared to post only) and through T-type VGCCs.

The strength of GABAergic synapses depends upon both the conductance of the channel and on the driving force for ions flowing through the channel. Because we recorded in a $\mathrm{HCO}_{3}^{-}$-free medium, $E_{\mathrm{GABA}} \approx E_{\mathrm{C}}$; thus the driving force through the $\mathrm{GABA}_{\mathrm{A}}$ receptors was largely determined by $E_{\mathrm{Cl}}$. We found that neither the channel conductance nor the $\mathrm{Cl}^{-}$driving force alone could predict the relationship between GPSC amplitude and $\mathrm{Ca}^{2+}$ influx. This indicates that it is the combination of these properties of GABAergic transmission that is important in regulating $\mathrm{Ca}^{2+}$ influx during STDP induction.

At glutamatergic synapses, positive and negative spike-timing intervals lead to long-term potentiation and depression, respectively, resulting in an asymmetric spike-timing window (Markram et al., 1997; Bi and Poo, 1998, 2001; Debanne et al., 1998; Zhang et al., 1998). In contrast, the spike-timing window for GABAergic synapses is symmetric, with coincident activity (within $\pm 15 \mathrm{~ms}$ ) resulting in decreased inhibition, independent of the spike-timing order (Woodin et al., 2003). This likely accounts for the nonsignificant differences in $\mathrm{Ca}^{2+}$-influx between positive and negative spike-timing intervals. However, the present study only examined spike-timing intervals of $<5 \mathrm{~ms}$; whether the results are similar for intervals $>5$ ms remains to be determined.

We identified a significant difference in the resting membrane potentials between depolarizing and hyperpolarizing synapses. This difference may result from our ability to identify GABAergic synapses where $E_{\mathrm{Cl}}$ sits close to the resting membrane potential; if $E_{\mathrm{Cl}} \approx$ resting membrane potential there would be no driving force for $\mathrm{Cl}^{-}$and thus we did not characterize a synapse electrophysiologically. This may have biased our selection of synapses for those with larger driving forces; depolarizing synapses would be more likely to be found onto neurons with relatively hyperpolarized resting membrane potentials, and hyperpolarizing synapses would be common onto neurons with relatively depolarized resting membrane potentials.

Following acute neuronal trauma (van den Pol et al., 1996; Toyoda et al., 2003), oxygen-glucose deprivation (Galeffi et al., 2004), and seizure activity (Galanopoulou, 2007), there is a depolarization of $E_{\mathrm{Cl}}$ which renders GABAergic transmission depolarizing (Fiumelli and Woodin, 2007; Kahle et al., 2008). Based on our present results, this switch in the polarity of GABAergic transmission increases the amount of $\mathrm{Ca}^{2+}$ influx during subsequent neuronal activity. In fact, the magnitude of the $E_{\mathrm{Cl}}$ depolarization following neuronal insults is so large it often renders GABAergic transmission excitatory (Kahle et al., 2008), which should produce even larger $\mathrm{Ca}^{2+}$ influxes than those observed in the present study. This may be particularly relevant given that the large $\mathrm{Ca}^{2+}$ influxes resulting from neuronal injury contribute to cell death (Bano and Nicotera, 2007).

Taken together, a model emerges where postsynaptic $\mathrm{Ca}^{2+}$ influx is required for STDP induction at GABAergic synapses, and where the magnitude of this influx is regulated by the GABAergic transmission itself. Further work will be need to elucidate both how the $\mathrm{Ca}^{2+}$ influx in turn regulates $E_{\mathrm{Cl}}$, and how the $\mathrm{Ca}^{2+}$ influx is regulated when GABAergic and glutamatergic STDP are induced simultaneously.

\section{ACKNOWLEDGMENTS}

This work was supported by a Natural Sciences and Engineering Research Council of Canada (NSERC) Discover Grant to Melanie A Woodin, and a NSERC Fellowship to Trevor Balena. 


\section{REFERENCES}

Balena, T., and Woodin, M. A. (2008). Coincident pre- and postsynaptic activity downregulates NKCC1 to hyperpolarize $\mathrm{E}(\mathrm{Cl})$ during development. Eur. J. Neurosci. 27, 2402-2412.

Balena, T., and Woodin, M. A. (2009). "Calcium dynamics underlying the induction of inhibitory synaptic plasticity," in Proceedings of the Society for NeuroscienceAnnualMeeting (Chicago, Illinois), 41.16.

Bano, D., and Nicotera, P. (2007). Ca2+ signals and neuronal death in brain ischemia. Stroke 38, 674-676.

Bi, G., and Poo, M., 2001. Synaptic modification by correlated activity: Hebb's postulate revisited. Annu Rev Neurosci. 24, 139-166.

Bi, G. Q., and Poo, M. M. (1998). Synaptic modifications in cultured hippocampal neurons: dependence on spike timing, synaptic strength, and postsynaptic cell type. J. Neurosci. 18, 10464-10472.

Blaesse, P., Airaksinen, M. S., Rivera, C., and Kaila, K. (2009). Cation-chloride cotransporters and neuronal function. Neuron 61, 820-838.

Cobb, S. R., Buhl, E. H., Halasy, K., Paulsen, O., and Somogyi, P. (1995). Synchronization of neuronal activity in hippocampus by individual GABAergic interneurons. Nature 378, 75-78.

Dan, Y., and Poo, M. M. (2006). Spike timing-dependent plasticity: from synapse to perception. Physiol. Rev. 86, 1033-1048.

Debanne, D., Gahwiler, B. H., and Thompson, S. M. (1998). Long-term synaptic plasticity between pairs of individual CA3 pyramidal cells in rat hippocampal slice cultures. J. Physiol. (Lond.) 507(Pt 1), 237-247.

Di Cristo, G., Wu, C., Chattopadhyaya, B., Ango, F., Knott, G., Welker,E., Svoboda, K., and Huang,Z.J. (2004). Subcellular domain-restricted GABAergic innervation in primary visual cortex in the absence of sensory and thalamic inputs. Nat. Neurosci. 7, 1184-1186.
Dzhala, V. I., Talos, D. M., Sdrulla, D. A., Brumback, A. C., Mathews, G. C., Benke, T. A., Delpire, E., Jensen, F. E., and Staley, K. J. (2005). NKCC1 transporter facilitates seizures in the developing brain. Nat. Med. 11, 1205-1213.

Fiumelli, H., and Woodin, M. A. (2007). Role of activity-dependent regulation of neuronal chloride homeostasis in development. Curr. Opin. Neurobiol. $17,81-86$.

Freund, T. F., and Buzsaki, G. (1996). Interneurons of the hippocampus. Hippocampus 6, 347-470.

Galanopoulou,A.S.(2007).Developmental patterns in the regulation of chloride homeostasis and GABA(A) receptor signaling by seizures. Epilepsia 48(Suppl. 5), 14-18.

Galeffi, F., Sah, R., Pond, B. B., George, A., and Schwartz-Bloom, R. D. (2004). Changes in intracellular chloride after oxygen-glucose deprivation of the adult hippocampal slice: effect of diazepam. J. Neurosci. 24, 4478-4488.

Gulledge, A. T., and Stuart, G. J. (2003). Excitatory actions of GABA in the cortex. Neuron 37, 299-309.

Huang, Z. J. (2006). Subcellular organization of GABAergic synapses: role of ankyrins and L1 cell adhesion molecules. Nat. Neurosci. 9, 163-166.

Kahle, K. T., Staley, K. J., Nahed, B. V., Gamba, G., Hebert, S. C., Lifton, R. P., and Mount, D. B. (2008). Roles of the cation-chloride cotransporters in neurological disease. Nat. Clin. Pract. Neurol. 4, 490-503.

Kaila, K. (1994). Ionic basis of GABAA receptor channel function in the nervous system. Prog. Neurobiol. 42, 489-537.

Kaila,K., andVoipio,J.(1987). Postsynaptic fall in intracellular $\mathrm{pH}$ induced by GABA-activated bicarbonate conductance. Nature 330, 163-165.

Kaila, K., Voipio, J., Paalasmaa, P., Pasternack, M., and Deisz, R. A. (1993). The role of bicarbonate in GABAA receptor-mediated IPSPs of rat neocortical neurones. J. Physiol. 464, 273-289.
Kano, M. (1994). Calcium-induced longlasting potentiation of GABAergic currents in cerebellar Purkinje cells. Jpn. J. Physiol. 44(Suppl. 2), S131-S136.

Magee, J. C., Christofi, G., Miyakawa, H., Christie, B., Lasser-Ross, N., and Johnston, D. (1995). Subthreshold synaptic activation of voltage-gated $\mathrm{Ca} 2+$ channels mediates a localized $\mathrm{Ca} 2+$ influx into the dendrites of hippocampal pyramidal neurons. J. Neurophysiol. 74, 1335-1342.

Markram, H., Lubke, J., Frotscher, M. and Sakmann, B. (1997). Regulation of synaptic efficacy by coincidence of postsynaptic APs and EPSPs. Science 275, 213-215.

Nishiyama, M., Hong, K., Mikoshiba, K., Poo, M. M., and Kato, K. (2000). Calcium stores regulate the polarity and input specificity of synaptic modification. Nature 408, 584-588.

Ormond, J., and Woodin, M. A. (2009). Disinhibition mediates a form of hippocampal long-term potentiation in area CA1. PLoS ONE 4, e7224. doi:10.1371/journal.pone.0007224.

Perez-Reyes, E., and Lory, P. (2006) Molecular biology of T-type calcium channels. CNS Neurol. Disord. Drug Targets 5, 605-609.

Rivera, C., Voipio, J., Payne, J. A. Ruusuvuori, E., Lahtinen, H., Lamsa, K., Pirvola, U., Saarma, M., and Kaila, K. (1999). The K+/Cl- co-transporter KCC2 renders GABA hyperpolarizing during neuronal maturation. Nature 397, 251-255.

Saraga, F., Balena, T., Wolansky, T. Dickson, C. T., and Woodin, M. A. (2008). Inhibitory synaptic plasticity regulates pyramidal neuron spiking in the rodent hippocampus. Neuroscience 155, 64-75.

Toyoda, H., Ohno, K., Yamada, J., Ikeda M., Okabe, A., Sato, K., Hashimoto, K., and Fukuda, A. (2003). Induction of NMDA and GABAA receptormediated $\mathrm{Ca} 2+$ oscillations with $\mathrm{KCC} 2$ mRNA downregulation in injured facial motoneurons. J. Neurophysiol. 89, 1353-1362. van den Pol,A. N., Obrietan, K., and Chen, G. (1996). Excitatory actions of GABA after neuronal trauma. J. Neurosci. 16, 4283-4292.

Woodin, M. A., Ganguly, K., and Poo, M. M. (2003). Coincident pre- and postsynaptic activity modifies GABAergic synapses by postsynaptic changes in Cl- transporter activity. Neuron 39 , 807-820.

Xu, C., Zhao, M. X., Poo, M. M., and Zhang, X. H. (2008). GABA(B) receptor activation mediates frequencydependent plasticity of developing GABAergic synapses. Nat. Neurosci. 11, 1410-1418.

Yamada, J., Okabe, A., Toyoda, H., Kilb, W., Luhmann, H. J., and Fukuda, A. (2004). Cl- uptake promoting depolarizing GABA actions in immature rat neocortical neurones is mediated by NKCC1. J. Physiol. 557, 829-841.

Zhang, L. I., Tao, H.W., Holt, C. E., Harris, W. A., and Poo, M. (1998). A critical window for cooperation and competition among developing retinotectal synapses. Nature 395, 37-44.

Conflict of Interest Statement: The authors declare that the research was conducted in the absence of any commercial or financial relationships that could be construed as a potential conflict of interest.

Received: 04 February 2010; paper pending published: 22 February 2010; accepted: 20 May 2010; published online: 28 June 2010.

Citation: Balena T, Acton BA and Woodin MA (2010) GABAergic synaptic transmission regulates calcium influx during spike-timing dependent plasticity. Front. Syn. Neurosci. 2:16. doi: 10.3389/ fnsyn.2010.00016

Copyright (C) 2010 Balena, Acton and Woodin. This is an open-access article subject to an exclusive license agreement between the authors and the Frontiers Research Foundation, which permits unrestricted use, distribution, and reproduction in any medium, provided the original authors and source are credited. 